\title{
ZERO-ONE LAWS FOR STABLE MEASURES
}

\author{
R.M.DUDLEY ${ }^{1}$ AND MAREK KANTER
}

ABSTRACT. For any stable measure $\mu$ on a vector space, every measurable linear subspace has measure 0 or 1 .

1. Introduction. It is known that for any Gaussian probability measure, a linear subspace has measure 0 or 1 . This result has been extended to additive subgroups by Kallianpur [2]. Here we extend the zero-one law in a different direction, replacing "Gaussian" by "stable". We begin with some definitions.

Definition. Let $S$ be a vector space over $R$ and let $\mathcal{S}$ be a $\sigma$-algebra of subsets of $S$. We call $(S, \mathcal{S})$ a measurable vector space iff both the following hold:

(a) addition is jointly measurable from $S \times S$ into $S$,

(b) scalar multiplication is jointly measurable from $\mathbf{R} \times S$ into $S$, for completed Lebesgue measure $\lambda$ on $\mathbf{R}$.

Let $S$ be a topological vector space and let $\mathcal{T}$ be the $\sigma$-algebra of Borel sets (generated by the open sets). Then if $S$ is metrizable and separable, $(S, \mathcal{T})$ is a measurable vector space, but it need not be so in general.

If $(S, \delta)$ is a measurable vector space and $\mu$ and $\nu$ are finite, countably additive measures on $\mathcal{S}$, then we have the convolution $\mu * \nu$ defined as usual by

$$
(\mu * \nu)(A)=(\mu \times \nu)\{\langle x, y\rangle: x+y \in A\} \text {. }
$$

For any ( $S$-valued) random-variable $Z$, let its probability distribution (law), defined on $\mathcal{S}$, be denoted by $\mathcal{}(Z)$.

Given any vector space $S$ and $c \in \mathbf{R}$, let $m_{c}(x) \equiv c x$ for all $x \in S$, and $\theta_{s}(x)=x+s$ for any $s \in S$.

Definition. Given a measurable vector space $(S, \mathcal{S})$, a probability

Received by the editors October 9, 1973.

A.MS (MOS) subject classifications (1970). Prim ary 60B 15; Secondary 60E05, $60 \mathrm{~F} 20,28 \mathrm{~A} 40$.

Key words and phrases. Stable measure, strictly stable, zero-one law, measurable vector space.

1 This research was partially supported by National Science Foundation Grant GP-29072. 
measure $\mu$ on $\mathcal{S}$ will be called stable iff for any $A>0$ and $B>0$, and independent random variables $X$ and $Y$ with distribution $\mu$, there is a $C>0$ and an $s \in S$ such that

$$
\begin{gathered}
\mathcal{L}(C(A X+B Y)+s)=\mu, \text { i.e. } \\
{\left[\left(\mu \circ m_{A}^{-1}\right) *\left(\mu \circ m_{B}^{-1}\right)\right] \circ m_{C}^{-1}=\mu \circ \theta_{-s}^{-1}}
\end{gathered}
$$

Definition. We say $\mu$ is strictly stable if we can always take $s=0$ in (1). We call $\mu$ symmetric iff $\mu(-E)=\mu(E)$ for all $E \in S$. We say $\mu$ is stable of index $\gamma$ if we can always take $C=C_{\gamma}(A, B) \equiv\left(A^{\gamma}+B^{\gamma}\right)^{-1 / \gamma}$.

$A$ random variable will be called stable iff its distribution is stable, and likewise for symmetry, strict stability, and the index.

The zero-one law (Theorem 6 and corollary) and its proof depend only on the above definitions. The following material, however, will help to clarify the meaning of stability in infinite-dimensional spaces.

If $S=\mathbf{R}$, then, as is well known (cf. Loève [4, pp. 326-328]) every stable $\mu$ has some index $\gamma \in(0,2]$, and the characteristic functions of stable laws of index $\gamma$ are of the form $e^{\phi(u)}$ where

$$
\begin{aligned}
\phi(u) & =i a u-b|u|^{\gamma}\{1+i c(\operatorname{sgn} u) \tan (\pi \gamma / 2)\}, & & \gamma \neq 1 ; \\
& =i a u-b|u|\left\{1+i c(\operatorname{sgn} u) 2 \pi^{-1} \log |u|\right\}, & & \gamma=1,
\end{aligned}
$$

where $a \in \mathbf{R}, b>0,|c| \leq 1, \gamma \in(0,2]$.

Simple calculations show that a stable $\mu$ with characteristic function having logarithm (2) is strictly stable iff $\alpha=0$ for $\gamma \neq 1$, while for $\gamma=1$ we have instead $c=0$. Also, $\mu$ is symmetric iff $\alpha=c=0 . \mu$ has a finite mean iff $\gamma>1$ (Feller [1, Vol. II., Theorem 1, p. 576]). Then the mean is 0 iff $\mu$ is strictly stable. Thus a strictly stable law may be called centered stable.

Theorem 1. Let $(S, \mathcal{S})$ be a measurable vector space and $\mu$ a symmetric stable measure on it. Then $\mu \circ \theta_{2 s}^{-1}=\mu$ for every $s$ appearing in (1).

Before proving this we note that in many cases it implies $s=0$, i.e. $\mu$ is strictly stable. For example if $S$ is a complete separable metric linear space and $\mathcal{S}$ is the Borel sets, $s$ must be 0 . To prove that every symmetric stable $\mu$ is strictly stable it would suffice to show that $\mu \circ \theta_{2 s}^{-1}=\mu$ implies $\mu \circ \theta_{s}^{-1}=\mu$, but we do not know whether this is true in general.

If $\mathcal{S}$ is a "degenerate" $\sigma$-algebra, e.g. if it is the smallest $\sigma$-algebra for which one linear form $\phi$ is measurable, and $S$ is more than one-dimensional, there exist $s \neq 0$ and $\mu$ such that $\mu \circ \theta_{u s}^{-1}=u$ for all $u \in \mathbf{R}$.

Proof. Suppose (1) holds for some $s$. Then in the notation of the definition of stability, and letting $\mathcal{L}(Z)$ denote the distribution of $Z$, we have 


$$
\mathcal{L}(X-s)=\mathscr{L}(C(A X+B Y))=\mathfrak{L}(-C(A X+B Y))=\mathfrak{L}(-X+s)=\mathfrak{L}(X+s),
$$

so the conclusion follows.

If $\mu$ is a stable law on a measurable vector space $(S, \mathcal{S})$ and $\phi$ is an $\mathcal{S}$-measurable linear form on $S$, then $\mu \circ \phi^{-1}$ is clearly stable on $R$. If $\mu$ has index $\gamma$, so does $\mu \circ \phi^{-1}$. If $\mu$ is strictly stable or symmetric, $\mu \circ \phi^{-1}$ has the same property.

It is known that on a complete metric linear space, any Borel measurable or even universally measurable linear form is continuous (theorems of Banach and Douady; cf. L. Schwartz [5, Lemme 2]). There exist such spaces, e.g. $L^{p}([0,1], \lambda)$ for $0 \leq p<1$, without any nonzero continuous linear forms, hence without nonzero measurable linear forms. On the other hand if $X_{n}$ are independent strictly stable real random variables of the same index $\gamma$ and $f_{n}$ are functions in $L^{p}$ with $\int\left|f_{n}\right|^{p} d \lambda \rightarrow 0$ fast enough as $n \rightarrow \infty$, then $\Sigma X_{n} f_{n}$ almost surely converges to an $L^{p}$-valued random variable whose distribution is clearly strictly stable of index $\gamma$. Thus stable measures on infinite dimensional spaces cannot always be treated in terms of characteristic functions nor measurable linear forms.

2. Linear forms. Let $S$ be a real vector space and $T$ a vector space of linear forms on $S$. Let $\delta(T)$ be the smallest $\sigma$-algebra for which all members of $T$ are measurable.

Theorem 2. Let $S$ be a vector space and $F$ a vector space of linear forms on $S$. Then $(S, S(F))$ is a measurable vector space.

Let $\mu$ be a probability measure on $\mathcal{S}(F)$. Then $\mu$ is strictly stable iff $\mu \circ t^{-1}$ is strictly stable for all $t \in F$.

Proof. To show that $(S, S(F))$ is a measurable vector space it is enough to show that for each $f \in F$, the maps $\langle s, t\rangle \rightarrow f(s+t)$ and $\langle x, s\rangle \rightarrow f(x s)$ are jointly measurable, which they clearly are.

As to the stability part, "only if" is clear, as above. To prove "if", suppose each $\mu \circ t^{-1}$ is strictly stable on $\mathbf{R}$. Let $\gamma(t)$ denote the index of $t$, i.e. $\mu \circ t^{-1}$. This is uniquely determined unless $\mu \circ t^{-1}$ is an atom at 0 , and then $t$ is stable of every index; we define $\gamma(t)=2$ in this case.

For any $d \in(0,2]$ let $F_{d}=\{t \in F: \gamma(t) \geq d\}$. Clearly $\gamma(t)=\gamma(u t)$ for any real $u \neq 0$. Hence if $t \in F_{d}$ then $u t \in F_{d}$ for all real $u$.

Suppose $t \in F_{d}$ and $\tau \in F_{d}$. Let $X_{1}, X_{2}, \cdots$ be independent with distribution $\mu$, and $S_{n}=X_{1}+\cdots+X_{n}$. Then $\mathcal{L}\left(t\left(S_{n}\right) / n^{1 / \gamma(t)}\right)=\mathcal{L}\left(t\left(X_{1}\right)\right)$, and likewise for $\tau$. 
For any $\delta<d$, we have $(t+\tau)\left(\mathrm{S}_{n}\right) / n^{1 / \delta} \rightarrow 0$ in probability as $n \rightarrow \infty$. Thus either $\gamma(t+\tau) \geq d$ or $t+\tau=0$ a.s. $(\mu)$, in which case we have set $\gamma(t+\tau)=2 \geq d$. Hence $F_{d}$ is a linear subspace of $F$.

For any two-dimensional subspace $A$ of $F, \gamma_{A} \equiv\{\gamma(t): t \neq 0, t \in A\}$ contains at most two points, since otherwise for some $t, \tau \in A, \gamma(t+\tau)<$ $\gamma(t)<\gamma(\tau)$, contradicting the above for $d=\gamma(t)$.

Further, if $\gamma_{A}$ contains two points $\delta<\kappa$, we can only have $\gamma=\kappa$ on a 1 -dimensional subspace $B=F_{\kappa} \cap A$ of $A$. Let $t \in B, t \neq 0$, and $t(n) \in A \sim$ $B, t(n) \rightarrow t$ in the usual topology of a plane. Then for any $u \in \mathbf{R}$,

$$
\lim _{n \rightarrow \infty}\left|E e^{i u t(n)}\right|^{2}=\left|E e^{i u t}\right|^{2} \text {. }
$$

From (2) above we get

$$
\left|E e^{i u t(n)}\right|^{2}=\exp \left(-c(n)|u|^{\delta}\right)
$$

for some $c(n)>0$, so we must have $c(n) \rightarrow 0$ and $t=0$ a.s. $(\mu)$.

Since any two points of $F$ belong to some two-dimensional space, it follows that for some $\gamma \in(0,2]$, all $t \in F$ are strictly stable of index $\gamma$; this includes the possibility $t=0$ a.s.

Now we show $\mu$ is strictly stable of index $\gamma$.

Let $C=C_{\gamma}(A, B)$ and $\nu=\mathfrak{\complement}(C(A X+B Y))$ in (1). Since every $t$ is strictly stable of index $\gamma$, we have $\nu \circ t^{-1}=\mu \circ t^{-1}$, and hence $\int e^{i t} d \nu=$ $\int e^{i t} d \mu$.

$B y$ the uniqueness theorem for characteristic functions on finite-dimensional vector spaces, the joint distribution of any finite set $\left(t_{1}, \cdots, t_{n}\right) \subset F$ is the same for $\nu$ as for $\mu$. Now since $\nu$ and $\mu$ are defined on $\mathcal{S}(F)$, they are equal, i.e. (1) holds. Q.E.D.

Theorem 3. Let $S$ be a vector space and $F$ a vector space of linear forms on $S$, and $\mu$ a probability measure on $\delta(F)$. Then $\mu$ is symmetric iff $\mu \circ t^{-1}$ is symmetric for all $t \in F$; also $\mu$ is symmetric and stable iff $\mu \circ t^{-1}$ is symmetric and stable for all $t \in F$.

Proof. This is an easy application of the previous theorem and me thod of proof.

Definition. Given a real vector space $S$ and a vector space $F$ of linear forms on $S$, we say $(S, F)$ is a full pair iff every real linear form $\phi$ on $F$ can be written $\phi(f) \equiv f(s)$ for some $s=s_{\phi} \in S$.

An example of a full pair is $\left(\mathbf{R}^{T}, F\right)$ where $\mathbf{R}^{T}$ is the set of all real-valued functions on a set $T$ and $F$ is the set of finite linear combinations of coordinate evaluations. 
Theorem 4. Suppose $(S, F)$ is a full pair and $\mu$ is a probability measure on $\left(S, \mathfrak{S}(F)\right.$ ) such that $\mu \circ f^{-1}$ is stable for all $f \in F$. Then $\mu$ is stable of some index $\gamma \in(0,2]$, and if $\gamma \neq 1$ there is a unique $t \in S$ such that $\mu \circ \theta_{t}^{-1}$ is strictly stable.

Proof. Let $\mu^{-}(E) \equiv \mu(-E)$ and let $\nu=\mu * \mu^{-}$. Then for every $f \in F$, $\nu \circ f^{-1}$ is symmetrically stable. Thus by Theorems 2 and $3, \nu$ is symmetric and strictly stable of some index $\gamma \in(0,2]$. It follows that for each $f \in F$, $\mu \circ f^{-1}$ is stable of index $\gamma$.

For each $f \in F$ there is some real number $k(f)$ such that if $X$ and $Y$ are independent with distribution $\mu$, then for $C=C_{\gamma}(A, B)$,

$$
\mathcal{L}(f(C[A X+B Y])+k(f))=\mathcal{L}(f(X)) \text {. }
$$

Since a Borel probability measure on $\mathbf{R}$ cannot equal a translate of itself, $k($.$) must be a linear form on F$. By fullness there is some $s=s(A, B) \in S$ such that $k(f)=f(s)$ for all $f \in F$. As in the previous proof, it follows using characteristic functions that (1) holds, so $\mu$ is stable.

Now suppose $\gamma \neq 1$. For any $f \in F$, there is some unique number $\alpha$ such that $f(X)+\alpha$ is strictly stable, by the characterization of strictly stable laws in (2). If we let $t=2^{1 / \gamma} s(1,1) /\left(2-2^{1 / \gamma}\right)$, then we must have $\alpha=f(t)$ by (1) with $A=B=1$. Hence $f(X+t)$ is strictly stable. Thus by Theorem 2 , $\mu \circ \theta_{t}^{-1}$ is strictly stable. Q.E.D.

If $(S, F)$ is not a full pair, then at any rate there is a topology $\mathcal{T}(S)$ on $F$ such that $(F, \mathcal{T})$ is a topological vector space and a linear form $\phi$ on $F$ is $\mathcal{T}$-continuous iff $\phi(f) \equiv f(s)$ for some $s \in S$. The weakest such topology is the weakest topology making each $f \rightarrow f(s)$ continuous; there may be others, as with infinite-dimensional Banach spaces and their duals.

Now let $f_{n}$ be a sequence such that $f_{n} \rightarrow 0$ for $\mathcal{T}$. Then $f_{n} \rightarrow 0$ pointwise on $S$, so in (3) we must have $k\left(f_{n}\right) \rightarrow 0$. Hence $k$ is sequentially $\mathfrak{J}$ continuous.

Definition. We call $(S, F)$ a semifull pair iff every sequentially $\mathcal{T}(S)$ continuous linear form on $F$ is of the form $f \rightarrow f(s)$ for some $s \in S$.

For examples of semifull pairs, let $(F, \mathcal{U})$ be any metrizable linear space and $S$ the dual space of all continuous linear forms on $F$.

Theorem 5. = Theorem 4 with "full" replaced by "semifull".

3. The zero-one law for linear subspaces.

Lemma 1. Let $\mu$ be a strictly stable measure on $(S, \mathcal{S})$. Let $E$ be a $\mu$ completion measurable linear subspace of $S$. Let $E^{\prime}=\{x \mid x \in S, E-x / r$ is 
$\mu$ completion measurable for all rational numbers $r>0\}$. Then $E^{\prime}$ is completion measurable and $\mu\left(E^{\prime}\right)=1$.

Proof. For every rational $r>0$, there is a positive real number $t(r)$ such that $\mu \circ \mu \circ m_{r}^{-1}=\mu \circ m_{t(r)}^{-1}$ by (1). Now $E / t(r)=E$ is $\mu$ measurable, hence $E$ is $\mu \circ m_{t(r)}^{-1}$ measurable. So for every $r, \exists$ sets $F_{r}, G_{r}$ in $\mathcal{S}$ with $F_{r} \subset E \subset G_{r}$ and $\mu \circ m_{t(r)}^{-1}\left(G_{r} \sim F_{r}\right)=0$. Let $F=\bigcup F_{r}, G=\bigcap G_{r}$, then we still have $\mu \circ m_{t(r)}^{-1}(G \sim F)=0$ for all $r$.

We can write

$$
\begin{aligned}
\mu \circ m_{t(r)}^{-1}(G \sim F) & =\int_{S} \mu \circ m_{r}^{-1}((G-x) \sim(F-x)) d \mu(x) \\
& =\int_{S} \mu((G / r-x / r) \sim(F / r-x / r)) d \mu(x) .
\end{aligned}
$$

Let $C_{r, x}=(G / r-x / r) \sim(F / r-x / r)$. It follows that for all $r, \mu\{x \mid$ $\left.\mu\left(C_{r, x}\right)=0\right\}=1$. Define $E^{\prime \prime}=\left\{x \mid \mu\left(C_{r, x}\right)=0\right.$ for all rational $\left.r\right\}$ and conclude that $E^{\prime \prime}$ is in $\mathcal{S}$ and $\mu\left(E^{\prime \prime}\right)=1$. However, since $F / r-x / r \subset E / r-x / r \subset G / r$ - $x / r$, we conclude that $E^{\prime \prime} \subset E^{\prime}$ and that $E^{\prime}$ is $\mu$ completion measurable with $\mu\left(E^{\prime}\right)=1$. Q.E.D.

Definition. If $\mu$ is strictly stable, we shall say that $\mu$ is well behaved if for all $\alpha \in(0,1)$ there is a $\beta \in(0,1)$ with

$$
\left(\mu \circ m_{\alpha}^{-1}\right) *\left(\mu \circ m_{\beta}^{-1}\right)=\mu \text {. }
$$

It is easy to show that if $\mu$ is strictly stable of index $\gamma$ then it is well behaved.

Theorem 6. Let $\mu$ be strictly stable and well behaved. Let $E$ be a linear subspace of $S$, measurable for the completion of $\mu$. Then $\mu(E)=0$ or 1 .

Proof. Suppose $\mu(E)>0$. Take $\alpha$ and $\beta$ so that (4) is satisfied with a rational. Let $E^{\prime}$ be defined as in the last lemma. Suppose $x \in E^{\prime} \sim E$ with $\mu(E-x)>0$. Then

$$
\begin{aligned}
\mu(E-a x) & =\left[\left(\mu \circ m_{a}^{-1}\right) *\left(\mu \circ m_{\beta}^{-1}\right)\right](E-\alpha x) \\
& \geq\left(\mu \circ m_{\alpha}^{-1}\right)(E-\alpha x)\left(\mu \circ m_{\beta}^{-1}\right)(E)=\mu(E-x) \mu(E)>0 .
\end{aligned}
$$

So the cosets $E-a x$ are all disjoint with measure bounded away from zero, as $\alpha$ ranges over the rationals. This is a contradiction so we conclude that $\mu(E-x)=0$ for $x \in E^{\prime} \sim E$.

Now take $A>0$ and take $C=C(A, A)$ so that (1) holds with $A=B$. Letting $\delta=A C$, we have that (4) holds with $\alpha=\beta=\delta$. We compute

$$
\mu(E)=\left(\mu \circ m_{\delta}^{-1}\right) *\left(\mu \circ m_{\delta}^{-1}\right)(E) .
$$

By arguing as in the preceding lemma we can conclude that $E-x$ is $\mu \circ m_{\delta}^{-1}$ completion measurable for $\mu \circ m_{\delta}^{-1}$ almost all $x$ and we can write 


$$
\begin{aligned}
\mu(E) & =\int_{s} \mu((E-\delta x) / \delta) d \mu(x) \\
& =\int_{E^{\prime}} \mu(E-x) d \mu(x)=\int_{E} \mu(E-x) d \mu(x)=\mu(E)^{2} .
\end{aligned}
$$

Thus, $\mu(E)=0$ or 1 . Q.E.D.

Corollary. If $E$ is a linear subspace of $S$ with $E \in \mathcal{S}$, then for any stable measure $\mu$ we have $\mu(E)=0$ or 1 .

Proof. Define the measure $\bar{\mu}$ by setting $\bar{\mu}(G)=\mu(-G)$ for $G \in \mathcal{S}$. Define $\nu$, the symmetrization of $\mu$, by $\nu=\mu * \bar{\mu}$. It is clear that $\nu$ is strictly stable since for all $A, B>0, \bar{\mu}$ satisfies (1) with $-s$ substituted for $s$. Now $\nu(E) \geq \mu(E)^{2}$. If $\mu(E)>0<\nu(E+x), x \notin E$, let $T=\{\alpha: \nu(E+\alpha x) \geq \nu(E) \nu(E+x)>$ $0\}$. Then for any $A, B, C$ in (1), $C A$ and $C B$ are in $T$ as in Theorem 6 . Thus the set of ratios of elements of $T$ is infinite and so is $T$. So we conclude that $\nu(E)=1$ as in the end of Theorem 6. But

$$
\nu(E)=\int \mu(E+x) d \mu(x) \text {. }
$$

It follows that $\mu(E+x)=1$ for $\mu$ almost all $x$ in $S$. In particular $\mu(E+x)=1$ for at least one $x$ in $E$ since $\mu(E)>0$. Now $E=E+x$ for $x$ in $E$, so $\mu(E)=1$. Q.E.D.

We can extend Theorem 6 to sets $E$ in $\mathcal{S}$ which are only assumed to be rational linear subspaces of $S$ (i.e., if $x, y \in E$ then $r x+s y \in E$ for any rational $r, s)$.

Theorem 7. Suppose $\mu$ is stable of index $\gamma$, where $\gamma$ is rational. If $E$ is an $\mathcal{S}$ measurable rational linear subspace of $S$, then $\mu(E)=0$ or $\mu(E)=1$.

Proof. By arguing as in the corollary to Theorem 6 , we are reduced to considering the case when $\mu$ is symmetric and strictly stable. We assume $\gamma=p / q$ where $p$ and $q$ are integers. By Waring's theorem [3, p. 37] there exists a positive integer $k$ such that any positive integer $b$ can be written in the form $b=a_{1}^{p}+\cdots+a_{k}^{p}$, where $a_{1}, \cdots, a_{k}$ are nonnegative integers. Now consider the equation

$$
a^{p}=(a-1)^{p}+a_{1}^{p}+\cdots+a_{k}^{p} .
$$

For any positive integer $a,(5)$ has a positive solution in integers $a_{i}$. We define $\left.r_{0}=(a-1) / a\right)^{q}, r_{1}=\left(a_{1} / a\right)^{q}, \cdots, r_{k}=\left(a_{k} / a\right)^{q}$ and we let $F(a)=$ $\Sigma_{0}^{k} r_{i}$. We conclude that $F(a) \rightarrow 1$ as $a$ goes to infinity and hence the equation

$$
1=r_{0}^{\gamma}+r_{1}^{\gamma}+\cdots+r_{k}^{\gamma}
$$


has infinitely many rational solutions and $\Sigma_{0}^{k} r_{i}$ can assume infinitely many values (unless $\gamma=1$; then proceed as in The orem 6).

Now $\mu=\mu \circ m_{r_{0}}^{-1} * \cdots * \mu \circ m_{r_{k}}^{-1}$ for any solution of (6) and we compute

$$
\begin{aligned}
\mu\left(E+\left(r_{0}+\cdots+r_{k}\right) x\right) & \geq\left(\mu \circ m_{r_{0}}^{-1}\left(E+r_{0} x\right)\right) \cdots\left(\mu \circ m_{r_{k}}^{-1}\left(E+r_{k} x\right)\right) \\
& =(\mu(E+x))^{k+1} .
\end{aligned}
$$

If $x$ is not in $E$ and $\mu(E+x)>0$, this leads to a contradiction, so we conclude $\mu(E+x)=0$ for all $x \notin E E$. The same reasoning shows that $\mu \circ$ $m_{\alpha}^{-1}(E+x)=0$ for all $x \notin E E$ and $\alpha$ rational.

Let now $\alpha>0, \beta>0, \theta>0$ be real with $\alpha^{\gamma}+\beta^{\gamma}=\theta^{\gamma}$ and $\alpha$ rational. We claim that

$$
\mu \circ m_{\alpha}^{-1}(E) \mu \circ m_{\beta}^{-1}(E)=\mu \circ m_{\theta}^{-1}(E) .
$$

This will follow if we show that the $\left(\mu \circ m_{a}^{-1}\right) \times\left(\mu \circ m_{\beta}^{-1}\right)$ product measure of the set $\{(x, y) \mid x \notin E, y \notin E, x+y \in E\}$ is 0 . However, this product measure equals $\int_{E} c^{\mu} \circ m_{a}^{-1}(E-y) d \mu \circ m_{\beta}^{-1}(y)$, which clearly is zero by the foregoing.

Now let $r_{1}, \cdots, r_{k}$ be any rational solution of (6) and define $\theta_{j}$ by $r_{1}^{\gamma}+\cdots+r_{j}^{\gamma}=\theta_{j}^{\gamma}$ for all $1 \leq j \leq k$. We have

$$
\mu \circ m_{\theta}^{-1}(E)=\mu \circ m_{r_{1}}^{-1}(E) \mu \circ m_{r_{2}}^{-1}(E) .
$$

Proceeding inductively by using (7), we conclude $\mu(E)=\Pi_{1 \leq j \leq k} \mu \circ m_{r_{j}}^{-1}(E)$. However, $\mu(E)=\mu \circ m_{r_{j}}^{-1}(E)$ for all $j$ so we conclude $\mu(E)=0$ or 1 . Q.E.D.

Acknowledgement. ${ }^{j}$ In the proof of Theorem 2 we had a useful discussion with D. Cohn and S. Portnoy.

\section{REFERENC ES}

1. W. Feller, An introduction to probability theory and its applications. Vol.II, 2nd ed., Wiley, New York, 1971. MR 42 \#5292.

2. G. Kallianpur, Zero-one laws for Gaussian processes, Trans. Amer. Math. Soc. 149 ( 1970), 199-211. MR 42 \#1200.

3. A. Hincin, Three pearls of number theory, Graylock Press, New York, 1952. MR 13, 724.

4. M. Loève, Probability theory. 3rd ed., Van Nostrand, Princeton, N.J., 1963. MR 34 \#3596.

5. L. Schwartz, Sur le théorème du graphe fermé, C. R. Acad. Sci. Paris Sér. A-B 263 (1966), A602-A605. MR 34 \#6494.

DEPARTMENT OF MATHEMATICS, MASSACHUSETTS INSTITUTE OF TECHNOLOGY, CAMBRIDGE, MASSACHUSETTS 02139

DEPARTMENT OF MATHEMATICS, SIR GEORGE WILLIAMS UNIVERSITY, MONTREAL, QUEBEC, CANADA 